\title{
STRAIN MEASUREMENTS OF RABBIT ACHILLES TENDONS BY ULTRASOUND
}

\author{
Po-Lin KuO, ${ }^{\dagger}$ PAI-ChI Li, ${ }^{*}$ Chia-Tung Shun ${ }^{*}$ and Jin-ShIn LaI ${ }^{\dagger}$ \\ ${ }^{\dagger}$ Department of Physical Medicine and Rehabilitation and ${ }^{*}$ Department of Pathology, National Taiwan University \\ Hospital, Taipei, Taiwan, ROC; and *Department of Electrical Engineering, National Taiwan University, \\ Taipei, Taiwan, ROC
}

(Received 12 November 1998; in final form 3 June 1999)

\begin{abstract}
Axial components of tendons' transverse strain fields were successfully measured in vitro by ultrasound. Achilles tendons of New Zealand white rabbits were used. Tendon inflammation was simulated by artificially inducing ischemia. Strain measurements were also correlated with conventional B-mode sonograms and histological micrographs. Results showed that strain measurements had reasonable agreement with histological examinations and provided better tissue differentiation than conventional B-mode imaging. For a given tendon, the strain values corresponding to different tissue layers were easily differentiated with an elastographic contrast-to-noise ratio ranging from 17 to $43 \mathrm{~dB}$. Such differences, however, were not always present in the sonograms. Therefore, ultrasonic strain imaging may be a useful tool for assessing tendon disorders during the rehabilitation process. (C) 1999 World Federation for Ultrasound in Medicine \& Biology.
\end{abstract}

Key Words: Elasticity imaging, Ultrasonic imaging, Tissue differentiation, Speckle tracking, Strain measurement, Tendon inflammation.

\section{INTRODUCTION}

One of the main tasks of tendons is to assist in concentrating the pull of the muscle on a small area of bone. The strength and stiffness of a tendon are provided by the cross-links between numerous collagen fibers that are parallel to its longitudinal axis. Because hematoma and inflammatory cell infiltration disrupt the arrangement of collagen fibers, the strength of a tendon tends to degrade when it is injured or inflamed (Mason and Allen 1941). The primary purpose of rehabilitation is to restore the mechanical functions of an injured or inflamed tendon. To monitor the tendon functions during the rehabilitation process, in vivo noninvasive methods such as ultrasound (US) are, thus, of great clinical importance.

As higher frequency transducers $(10 \mathrm{MHz}$ and above) became available for clinical use, applications using conventional B-mode imaging to image injured or inflamed tendons have gained broad interest. Compared to a normal tendon, thickness of an inflamed tendon increases at early inflammation stage due to hematoma

Address correspondence to: Dr. Jin-Shin Lai, Department of Physical Medicine and Rehabilitation, National Taiwan University Hospital, No.7, Chung-Shan South Road, Taipei, Taiwan 100, ROC. E-mail: jslai@ccms.ntu.edu.tw accumulation and inflammatory cell infiltration. Hence, the tendon thickness has been used to recognize morphological change associated with an inflamed tendon (Fornage 1995). However, as an injured or inflamed tendon progresses (favorably or unfavorably), the thickness does not change significantly (Holsbeeck and Introcaso 1991). Therefore, distinction among various recovery stages is difficult based only on the thickness of the tendon. In addition to the thickness, tendon abnormality may also be detected based on B-mode texture features (Fornage 1995; Holsbeeck and Introcaso 1991; Marcelis 1996). However, due to the low contrast between normal connective and granulation tissues and the subjective nature of image interpretation, success of such techniques has also been limited.

Another potential application of US in this area is elasticity imaging. Conventional B-mode imaging is used to produce pictures of anatomical structures. Ultrasonic elasticity imaging, on the other hand, is used to quantitatively assess the elastic properties of biological tissue. Such an imaging method typically involves externally applied forces and reconstruction of strain fields. Several research groups have published results on theories (Kallel and Bertrand 1996; Kallel et al. 1996, 1997, 1998; Ponnekanti et al. 1995; Skovoroda et al. 1994, 
1995; Sumi et al. 1995; Varghese and Ophir 1997a, 1997c), data acquisition protocols (Krouskop and Levison 1987; Lerner et al. 1990; Ophir et al. 1991; Sarvazyan 1997; Yamakoshi 1990), signal-processing techniques (Cespedes and Ophir 1993; Insana et al. 1996; Kaisar and Ophir 1997; O’Donnell et al. 1994; Varghese and Ophir 1996, 1997b; Varghese et al. 1996; Yeung et al. 1998), and preliminarily clinical investigation for elasticity imaging (Cespedes et al. 1993; Garra et al. 1997; Levison et al. 1995). Elasticity imaging of tendons, however, has not been explored.

Stress and strain are commonly used to describe the elastic properties of a material. Most biological soft tissues have nonlinear viscoelastic behavior and the constitutive equations specifying the stress-strain relationships are complex (Fung 1993). Under static deformation with small loading, however, a linear elastic model can be applied and the tensor of elastic moduli is derived by the generalized Hooke's law (Ponnekanti et al. 1995; Skovoroda et al. 1994). Therefore, the reconstruction of elasticity imaging can be carried out by an inverse problem approach (Kallel and Bertrand 1996; Ophir et al. 1997). Solving the inverse problem in elasticity imaging generally constitutes two steps. The first step involves estimating the strain fields from the US data acquired pre- and post-compression. The second step is to find the optimal distribution of elastic modulus given the estimated strain fields. The primary purpose of this study is in regard to the first step of elasticity imaging. Feasibility of estimating the strain fields of tendons using US was studied. Furthermore, the distribution of strain fields was compared to B-mode sonograms and correlated with histology.

\section{THEORY}

Deformation of a material due to an applied stress can be described by the strain. Specifically, the axial strain $\varepsilon$ is defined as:

$$
\varepsilon=\frac{\Delta z_{2}-\Delta z_{1}}{\Delta z},
$$

where $\Delta z_{1}$ is the displacement at depth $z, \Delta z_{2}$ denotes the displacement at depth $z+\Delta z$, and $\Delta z$ represents the spatial interval between these two depths (Ophir et al. 1991). One approach for displacement measurements is to estimate tissue motion by speckle tracking. In this paper, a baseband cross-correlation approach is employed (O'Donnell et al. 1994).

Speckle results from coherent interference from many scatterers within one sample volume and acts as image markers for motion tracking. Let $X_{1}(t)$ be the complex representation of the echo reflected from the object of interest and we have:

$$
X_{1}(t)=A\left(t-\tau_{1}\right) e^{j \omega\left(t-\tau_{1}\right)},
$$

where $A(t)$ is the envelope of the signal, $\omega$ is the angular frequency of the ultrasound carrier and $\tau_{1}$ is the roundtrip propagation time between the object and the transducer. With the application of an external force, the object moves, and the signal from the same object becomes:

$$
X_{2}(t)=A\left(t-\tau_{2}\right) e^{j \omega\left(t-\tau_{2}\right)},
$$

where $\tau_{2}$ is the new round-trip propagation time.

Let $B_{1}(t)$ and $B_{2}(t)$ denote the baseband representations of $X_{1}(t)$ and $X_{2}(t)$, and we have:

$$
B_{1}(t)=X_{1}(t) \times e^{-j \omega t}=A\left(t-\tau_{1}\right) e^{-j \omega \tau_{1}},
$$

and

$$
B_{2}(t)=X_{2}(t) \times e^{-j \omega t}=A\left(t-\tau_{2}\right) e^{-j \omega \tau_{2}} .
$$

The cross-correlation function of $B_{1}(t)$ and $B_{2}(t)$, thus, becomes:

$$
C(t)=\int_{-\infty}^{\infty} B_{1}(t+\tau) \times B_{2}{ }^{*}(\tau) d \tau,
$$

where $*$ denotes the complex conjugate. Setting $t=0$, we have:

$$
\begin{aligned}
C(0) & =\int_{-\infty}^{\infty} B_{1}(\tau) \times B_{2}^{*}(\tau) d \tau \\
& =\left[\int_{-\infty}^{\infty} A\left(\tau-\tau_{1}\right) \times A\left(\tau-\tau_{2}\right) d \tau\right] e^{j \omega\left(\tau_{2}-\tau_{1}\right)} .
\end{aligned}
$$

Hence,

$$
\tau_{2}-\tau_{1}=\frac{\angle C(0)}{\omega},
$$

where $\angle C(0)$ is the angle of the complex function $C(0)$. Assuming that the sound velocity is a constant $c$, the displacement becomes: 


$$
\Delta z=\frac{c \times\left(\tau_{1}-\tau_{2}\right)}{2} .
$$

Notice that the time delay is divided by two for round trip propagation.

After the displacement is obtained, the strain field can be estimated by using eqn (1).

\section{MATERIALS AND METHODS}

Eight Achilles tendons from four adult New Zealand white rabbits were used in this study. The animals were purchased from the National Animal Center and were housed in animal rooms with good environmental control. The room temperature was kept at $24 \pm 1^{\circ} \mathrm{C}$ under a 12-h light (07:00 to 19:00) and 12-h dark regimen. For each rabbit, one randomly selected hind limb was included in the Inflamed Group and the other hind limb was included in the Healthy Group. We have previously found that ischemia can induce tendon inflammation. Therefore, the tendons in the Inflamed Group were prepared based on the following procedures. First, general anesthesia was applied successfully with $5 \mathrm{mg}$ phenothiazine and $25 \mathrm{mg}$ ketamine per kg body weight intramuscularly (IM). Next, the skin overlying the Achilles tendon was shaved, scrubbed and anesthetized locally with $20 \mathrm{mg}$ lidocaine. A longitudinal incision was made lateral to the visible outline of the tendon. By blunt dissection, the Achilles tendons in the Inflamed group were then exposed, isolated from the adjoining tissue and tightened circumferentially on the rostral and caudal sides with nylon sutures. The rostral site was near the tendon and muscle belly junction. The caudal site was near the tendon and calcaneus junction. The sutures were tightened firmly to simulate tendon ischemia. The skin was then closed. No particular treatments were done to the tendons in the Healthy Group.

After the rabbits regained consciousness, they were housed one per standard rabbit cage and raised as normal. Food and water were available $a d l i b$. The use and care of animals were in accordance with the principles of the National Animal Center of ROC. We have found empirically that a period of 10 days is needed to ensure obvious inflammation for ischemic tendon after wound closure. At that time, Achilles tendons in both the Inflamed Group and the Healthy Group were harvested. The tendons were excised from the tendon-muscle junction proximally and the tendon-calcaneus junction distally. The size of tendon samples was about $20 \sim 30 \mathrm{~mm}$ long and $4 \sim 9 \mathrm{~mm}$ in diameter. After excision, each sample was then immediately immersed in the center of a gelatin-based phantom and was paired with its opposite side.

The image phantoms were constructed from agar

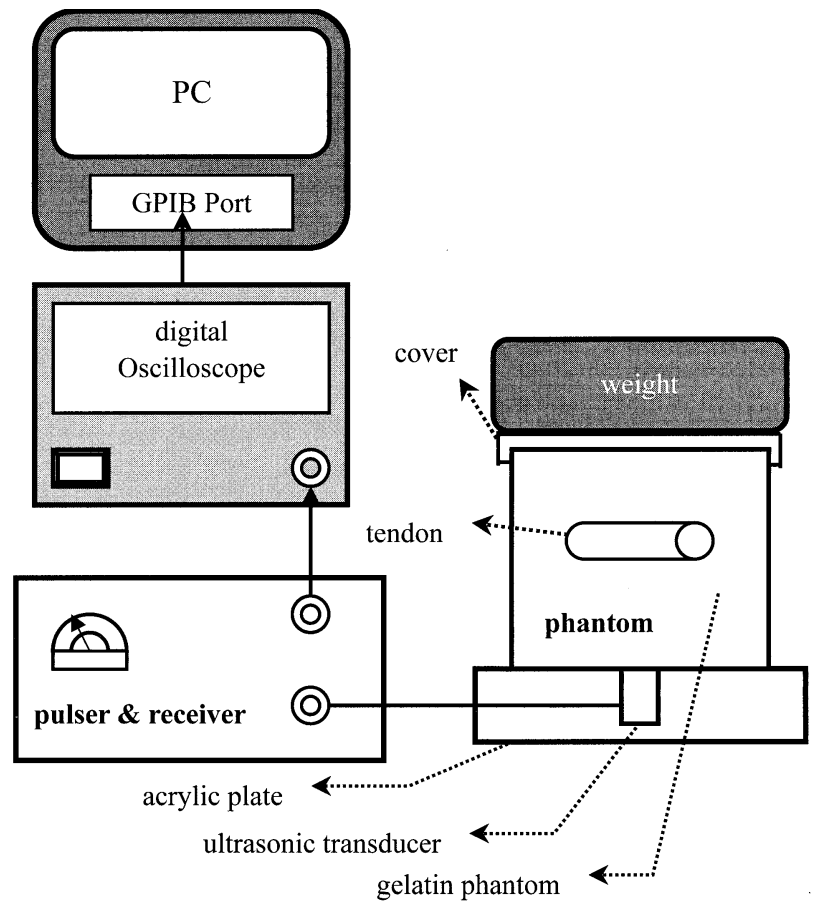

Fig. 1. Schematic diagram of the experimental setup.

powder (5\% by weight) with similar stiffness to each other. The phantoms were cylindrical, with a diameter of $114.5 \mathrm{~mm}$ and a length of $45.0 \mathrm{~mm}$. The agar powder was hydrated with deionized water and heated above its gel point to disperse the colloid, clarify the solution and release trapped gasses. The liquid gel solution was then cooled to $45^{\circ} \mathrm{C}$ and poured into a cylindrical mold kept in an icewater bath. When making the phantoms, the following steps were taken to keep the longitudinal axis of the tendon sample parallel to the surface of the phantom. First, gel solution was poured into the mold until a height of $20 \mathrm{~mm}$ was reached. Next, as the gel surface congealed, the tendon sample was put on the center of this surface and additional gel solution was poured into the remaining mold space. All the above procedures were performed on a leveled desk. After the completion of the above procedures, the phantoms were stored at $10^{\circ} \mathrm{C}$ and ultrasound data were collected within $12 \mathrm{~h}$.

A schematic representation of the strain measurement system is illustrated in Fig. 1. An unfocused, $5-\mathrm{MHz}$ circular transducer (Panametrics V110, Waltham, MA) with a diameter of $7.9 \mathrm{~mm}$ was attached to a hole in an acrylic plate with the direction of the US beam perpendicular to the plate. Note that the top of the phantom is defined as the weight side and the bottom is defined as the transducer side. The distance between the sample and the transducer was set at $20 \mathrm{~mm}$ to reduce near-field effects and to avoid transducer ring-down artifacts. Prior to each measurement, the acrylic base was 
leveled so that direction of the gravitational force applied at the top of the phantom was parallel to the direction of the axial displacement. The transducer was connected to an ultrasonic pulser/receiver (Panametrics 5072PR, Waltham, MA) for A-scan data acquisition. Maximum output power was used. Attenuation and damping settings, on the other hand, were adjusted for each measurement to achieve sufficient signal-to-noise ratios. A digital oscilloscope (Lecroy 9314L, Geneva, Switzerland) was used to digitize the received signal at $100 \mathrm{MHz}$.

Ultrasonic coupling gel was applied on the transducer face for efficient acoustic transmission. The phantom was positioned so that the tendon intersected with the center of the ultrasound beam. The phantom was compressed by adding weights to the top surface (i.e., the weight side). A $0.5-\mathrm{kg}$ weight was initially used to preload the phantom and to ensure good contact at the transducer-phantom boundary. After the phantom was preloaded, additional weights were applied with a $0.5-\mathrm{kg}$ increment. We have found that a $0.5-\mathrm{kg}$ increment was able to provide sufficient displacements measurable by the speckle-tracking technique and avoiding signal decorrelation resulting from changes in scatterer distribution. After each increment, returning echoes were sampled using a digital oscilloscope. The digitized A-scan signals were then transferred to a personal computer via a GPIB port for off-line signal processing.

The cross-sectional area of the weights was the same as that of the phantom. In other words, the top surface of the phantom was fully covered. Additionally, a plastic cover was placed between the weights and the phantom to ensure uniform external stress distribution at the top surface. The experiments were performed at room temperature.

The stress fields produced tendon displacements. Displacement relative to the bottom tendon boundary was defined as the relative displacement. For strain estimation, only the relative displacements between the top and the bottom tendon boundaries were required. Displacement of the entire tendon (i.e., bulk translation) was eliminated by signal alignment. As described previously (O'Donnell et al. 1994), aliasing results from phase differences greater than $\pm \pi$. In other words, aliasing occurs when the displacement is larger than $\lambda / 4(75 \mu \mathrm{m}$ at 5 $\mathrm{MHz}$ ), where $\lambda$ is the wavelength at the carrier frequency. It was confirmed that aliasing was not present in the data acquired in this study.

As demonstrated in Fig. 2, a moving window was used to select data from each data set for displacement calculation. The window size and the interval between consecutive windows were chosen based on the following criteria. First, the interval is limited by the window size and the overlap factor. Also, the interval needs to be sufficiently small (about $0.5 \sim 0.8 \mathrm{~mm}$ ) so that detailed

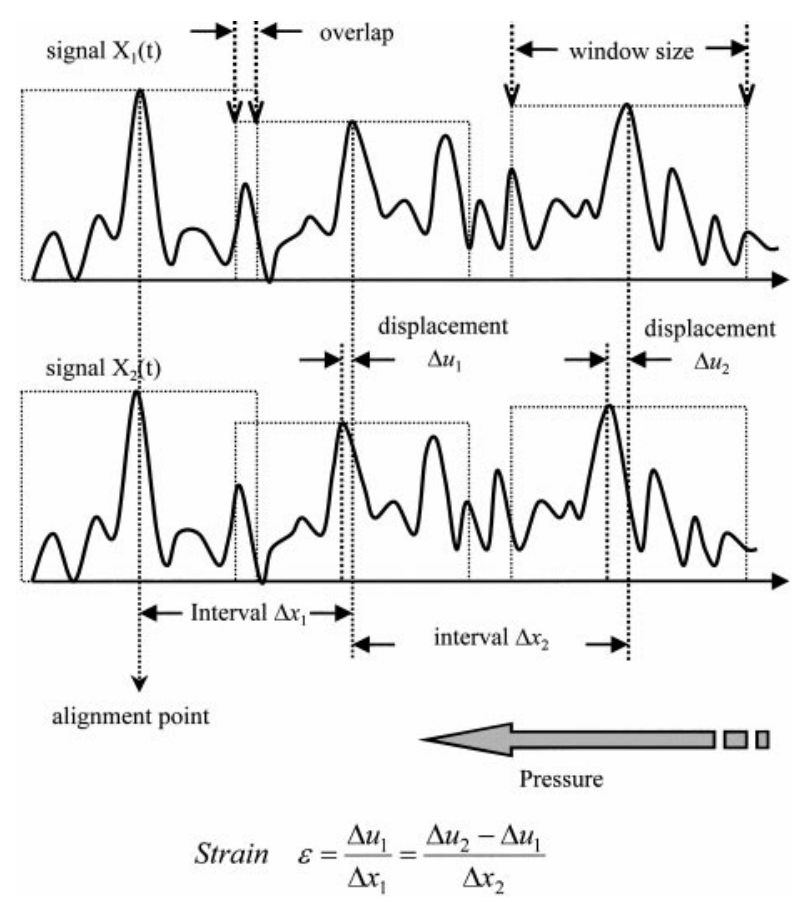

Fig. 2. Demonstration for strain estimation using speckle tracking. $X_{1}(t)$ and $X_{2}(t)$ are signals with and without incremental compression. The dotted rectangles represent moving windows for data selection.

strain variations can be adequately detected. The interval was fixed within each data set.

Second, selection of the window size is affected by several factors. In general, a large window size is desired to reduce strain estimation variance (Varghese and Ophir 1997a). As the window size increases, however, signal decorrelation deteriorates accuracy of displacement estimation (Cespedes and Ophir 1993; O’Donnell et al 1994; Varghese and Ophir 1997a). The decorrelation effect is more pronounced in the high strain region. Because the strain field may be nonuniform within the tendon, the desired window size may also vary at different window positions. Generally speaking, large windows provide low variances for small strains, whereas small windows are desired to minimize signal decorrelation in the presence of large strains (Varghese and Ophir 1998). For each window position, the window size was carefully chosen to achieve high correlation between the two data sets used for displacement estimation. The correlation coefficients were above 0.98 . In addition, the window size ensures that the estimated relative displacements increase monotonically as the positions are away from the lowest bound of tendon.

After A-scan data collection, the tendons were imaged by a commercial scanner (ATL HDI 3000, Bothell, WA) using a 128-channel linear transducer with a center frequency of $10 \mathrm{MHz}$. After the B-mode images were 


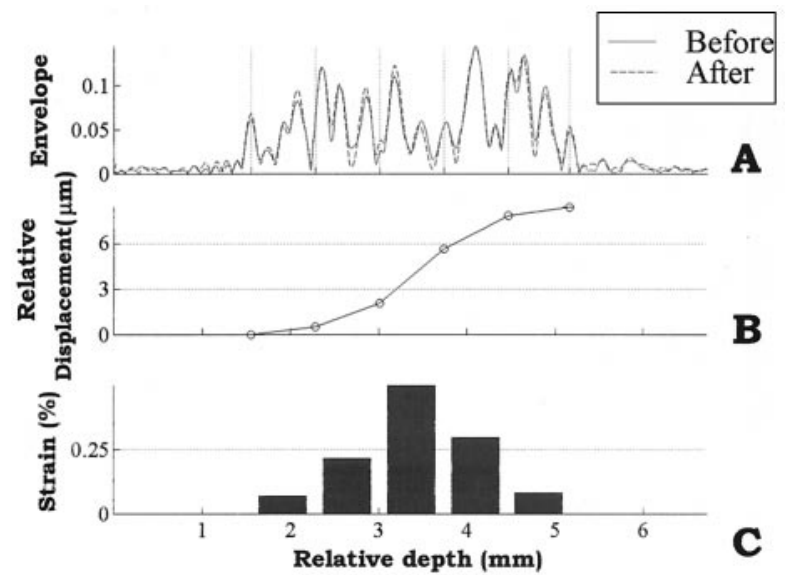

Fig. 3. Measured strain field for Sample 3H (Pair 3, healthy). (A) RF signals with and without incremental compression; (B) relative displacement; (C) strain field.

obtained, the tendons were then fixed in $10 \%$ neutral formalin and stained with routine hematoxylin and eosin $(\mathrm{H} \& \mathrm{E})$ before being examined by a light microscope (Olympus BH-2, Tokyo, Japan).

The sonograms and the corresponding estimated strain fields were evaluated with the same spatial orientation by three experienced clinicians. An elastographic contrast-to-noise ratio $\left(\mathrm{CNR}_{\mathrm{e}}\right)$ was used to quantify the ability to resolve regions with different strain values. $\mathrm{CNR}_{\mathrm{e}}$ is defined following the description in Bilgen and Insana (1997) and Varghese and Ophir (1998):

$$
C N R_{e}=\frac{2\left(s_{1}-s_{2}\right)^{2}}{\left(\sigma_{s 1}^{2}+\sigma_{s 2}^{2}\right)},
$$

where $s_{1}$ and $s_{2}$ represent mean strain values in the first and the second region, respectively. Similarly, $\sigma_{s 1}^{2}$ and $\sigma_{s 2}^{2}$ denote the strain variances in the respective region. The numerator in eqn (10) represents the strain contrast between the two different regions, and the denominator represents the average variance. In the following analysis, the $C N R_{e}$ is converted on a logarithmic scale shown below (Varghese and Ophir 1998):

$$
C N R_{e}(d B)=20 \log _{10}\left[\begin{array}{cc}
2\left(s_{1}-s_{2}\right)^{2} /\left(\sigma_{s 1}^{2}+\sigma_{s 2}^{2}\right) & ; s_{1} \neq s_{2} \\
1 & ; s_{1}=s_{2}
\end{array}\right]
$$

Finally, both the sonograms and the estimated strain fields were compared with the corresponding histology micrographs with correct spatial orientation. The length of a particular region on a micrograph or a sonogram was measured along the direction parallel to the ultrasound beam (i.e., the axial direction).

\section{RESULTS}

Results from four pairs of tendons were shown. Except for Pair 1, all samples in the Inflamed Group showed different histological results than the corresponding samples in the Healthy Group. The sample associated with Pair 1 in the Inflamed Group was found normal in histological examinations. This may be due to failure of ischemia simulation resulting from loosely tightened sutures. Nevertheless, the other tendons in the Inflamed Group (i.e., Pairs 2, 3 and 4) showed abnormalities such as proliferation of granulation tissue or tissue necrosis.

A typical example of strain reconstruction is shown in Fig. 3. Fig. 3A shows the alignment of two RF signals with and without the application of incremental weight. The larger amplitude signals were recognized as the signals of tendon and both data sets were aligned at the left tendon boundary. Note that most of the small-amplitude signals outside the tendon are not shown. Also note that all three panels have the same horizontal scale as in Fig. 3C and the depth is relative to an arbitrary starting point. The size of tendon is about $3.5 \mathrm{~mm}$ (from $1.5 \mathrm{~mm}$ to $5 \mathrm{~mm}$ ) in Fig. 3A. Displacements relative to the left tendon boundary are plotted in Fig. 3B. Using eqn (1) and the procedures previously outlined, strain distribution is reconstructed and shown in Fig. 3C.

Figure 4 shows $\mathrm{B}$-mode sonograms and estimated strain field of sample 2H (Pair 2, healthy). Figure 4A is
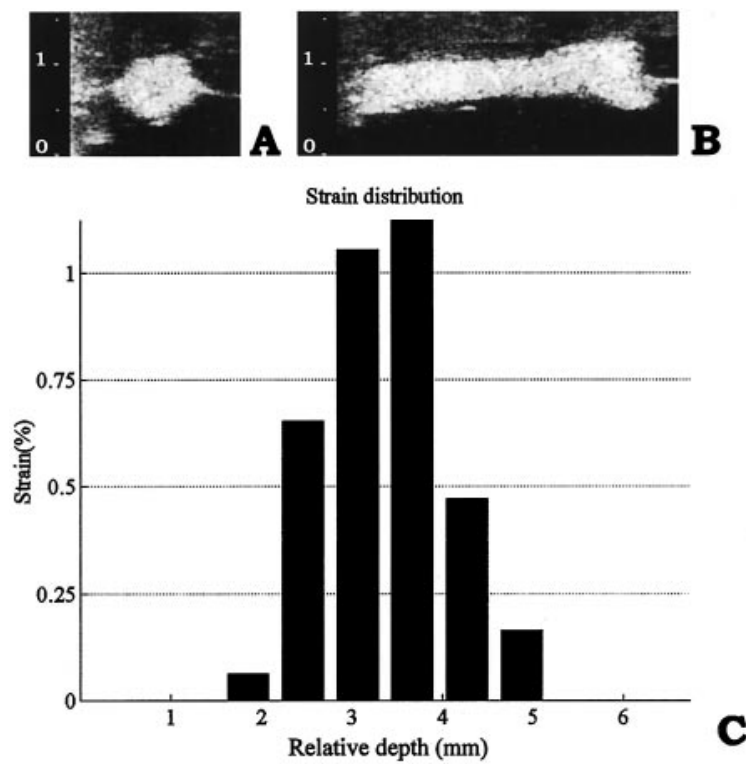

Fig. 4. B-mode sonograms and strain field for Sample 2H (Pair 2, healthy). (A) B-mode sonogram in the transverse view; (B) B-mode sonogram in the longitudinal view; (C) strain field. The interval between two tick marks on the left side of each sonogram is $5 \mathrm{~mm}$. 


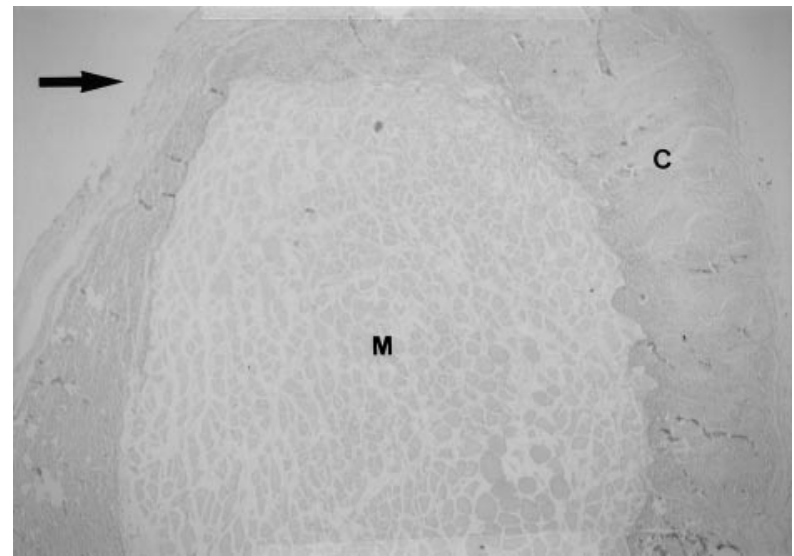

Fig. 5. Micrograph of Sample $2 \mathrm{H}$ (Pair 2, healthy). $\mathrm{M}=$ the muscle cells; $\mathrm{C}=$ bundles of collagen fibers. The black arrow represents the direction parallel to the ultrasound beam. (H\&E $20 \times)$.

the B-mode sonogram of this healthy tendon in the transverse view and Fig. 4B shows the longitudinal view. The tendon appears as a homogeneous, hyperechoic region in both views. Note that the sonograms and strain field in Fig. 4 are oriented in such a way that the bottom
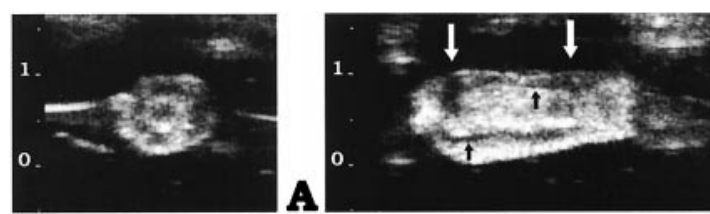

B

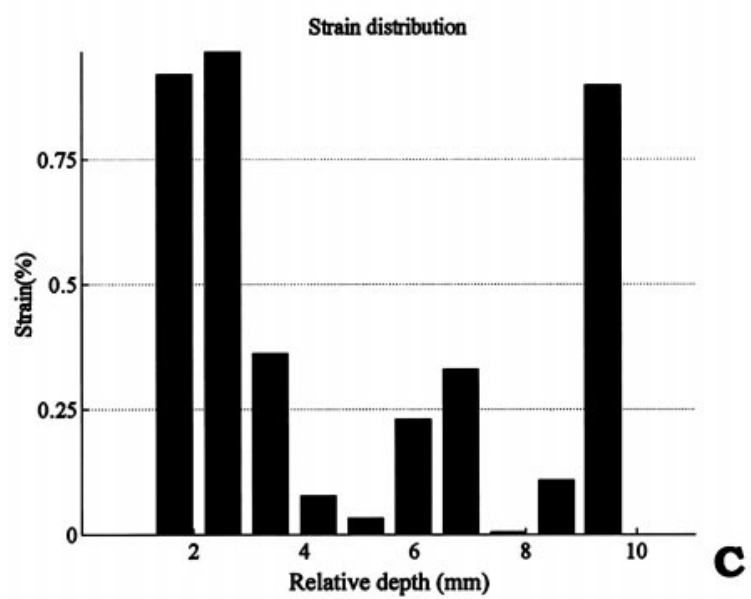

Fig. 6. B-mode sonograms and strain field for Sample 3I (Pair 3 , inflamed). (A) B-mode sonogram in the transverse view; (B) B-mode sonogram in the longitudinal view; (C) strain field. The white arrows in (B) highlight the suture artifacts. The black arrows in (B) point out the hypoechoic boundary between the outer and inner concentric regions. of sonograms and the left of strain field correspond to the transducer side of the phantom. The thickness of tendon estimated from Fig. $4 \mathrm{C}$ is similar to that estimated at the middle segment in Fig. 4B (about $4 \mathrm{~mm}$ ). Although the tendon appears homogeneous in B-mode, the transverse strain shown in Fig. $4 \mathrm{C}$ varies over a wide range. By using the region between $2.2 \mathrm{~mm}$ and $4.5 \mathrm{~mm}$ in Fig. 4C as a different tissue from that near the edges, the $\mathrm{CNR}_{\mathrm{e}}$ calculated by eqn (11) is about $20 \mathrm{~dB}$. The strain variations can be explained by the micrograph shown in Fig. 5. The micrograph shows that the specimen consists of muscle tissue in the center and bundles of collagen fibers spreading near the edges. Note that the central muscle tissue is about 4 times as thick as the peripheral collagen fiber layer. This ratio is consistent with that shown in the estimated strain field. Although the strain values are clearly different as shown in the Fig. 4C, the differences between muscle and bundles of collagen fibers are not detectable in B-mode. The other samples in the Healthy Group and Sample 1I (Pair 1, inflamed) exhibit similar results. The values of estimated $\mathrm{CNR}_{\mathrm{e}}$ range from $17 \mathrm{~dB}$ to $22 \mathrm{~dB}$ among these samples.

Similar comparisons were carried out for the samples in the Inflamed group. Figure 6 shows B-mode sonograms and strain field for Sample 3I (Pair 3, inflamed) using the same format as in Fig. 4. The sonograms in Fig. 6A and B show that the tendon has two concentric hyperechoic regions. As illustrated in Fig. 6C, the strain filed also has two distinct regions with low strain values in the middle and high ones near the edges. The thickness measured from Fig. 6A is consistent with that measured from Fig. 6C. For example, the thickness of the left high strain region in Fig. 6C and the bottom hyperechoic layer in Fig. 6A are both around $2 \mathrm{~mm}$. Likewise, the thickness for the middle low strain region

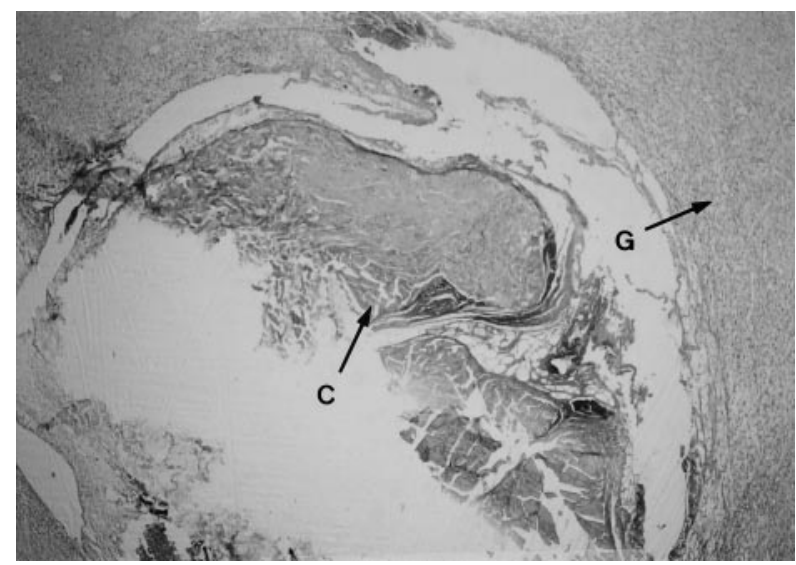

Fig. 7. Micrograph of Sample 3I (Pair 3, inflamed). C = bundles of collagen fibers; $\mathrm{G}=$ the circularly surrounding granulation tissue. (H\&E 20×). 

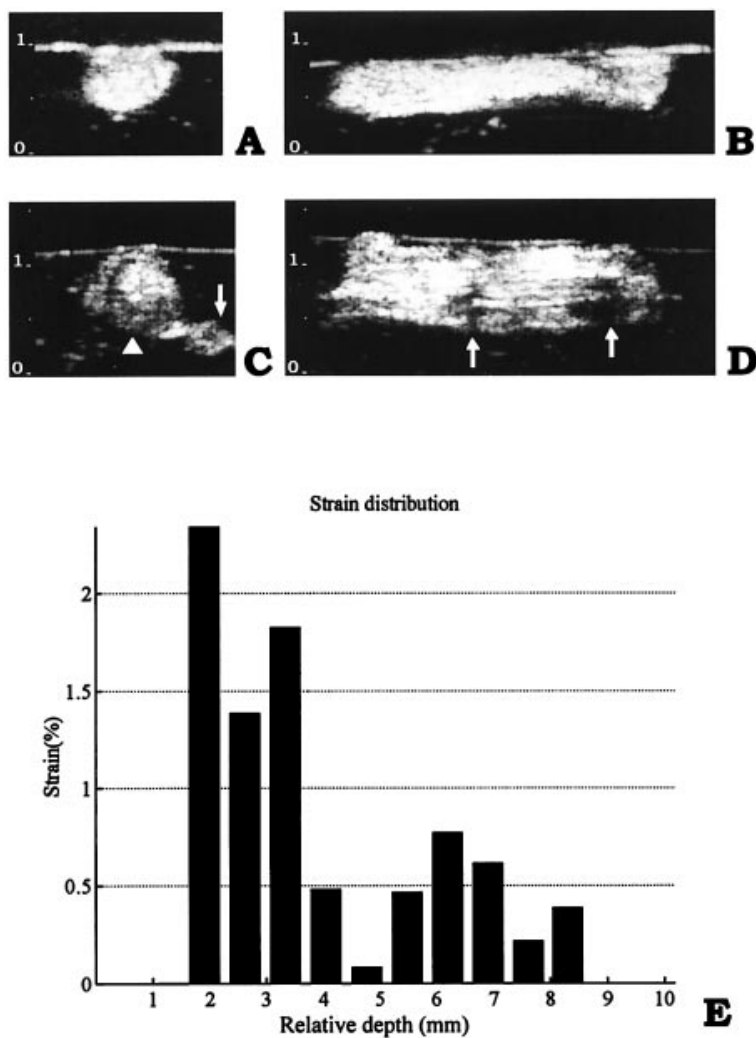

$\mathbf{E}$

Fig. 8. B-mode sonograms and strain fields for Samples $4 \mathrm{H}$ and 4I (Pair 4, healthy and inflamed). (A) and (B) are Sample 4H. (C) and (D) are for Sample 4I. (A) and (C) are sonograms in the transverse view. (B) and (D) are sonograms in the longitudinal view. The white arrow in (C) indicates tissue debris. The white triangle denotes the semilunar region with low brightness. The white arrows in (D) highlight the suture artifacts. (E) is the strain field of sample 4I.

(or the middle hyperechoic area) is around $6 \mathrm{~mm}$ and the thickness for the right high strain region (or the top hyperechoic layer) is around $1 \mathrm{~mm}$. The echo texture of these two hyperechoic regions on the sonograms was too similar for the evaluators to distinguish. On the contrary, the $\mathrm{CNR}_{\mathrm{e}}$ of these two regions in the estimated strain field is about $34 \mathrm{~dB}$. In other words, the estimated strain field provides better tissue characterization than conventional B-mode imaging in this particular example.

The micrograph shown in Fig. 7 confirms that these distinct regions correspond to different tissues. The inner part of the specimen corresponds to bundles of collagen fibers and the peripheral high-strain regions correspond to circularly proliferated granulation tissue.

Figure 8 compares a healthy sample (Sample $4 \mathrm{H}$ ) to the corresponding inflamed sample (Sample 4I). Figure $8 \mathrm{~A}$ and B show B-mode sonograms of Sample $4 \mathrm{H}$ and Fig. 8C and D for Sample 4I. Sonograms of the healthy tendon again appear as a homogeneous, hyperechoic

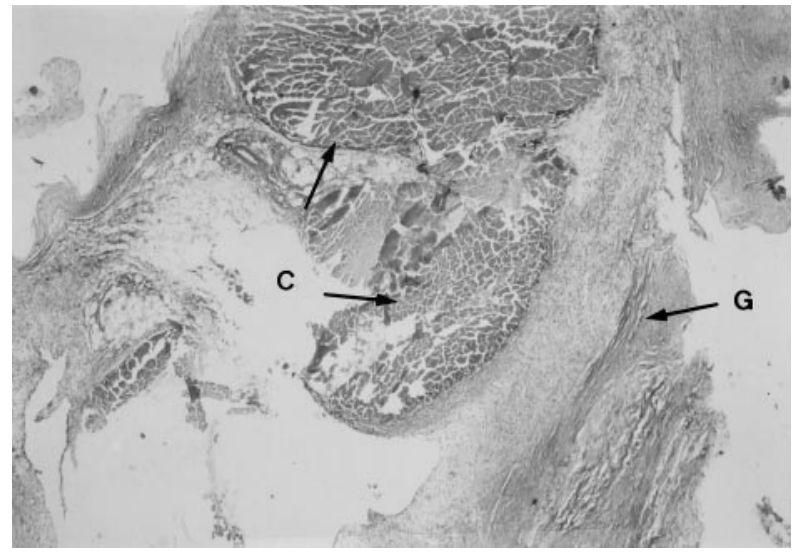

Fig. 9. Micrograph of Sample 4I (Pair 4, inflamed). C = bundles of collagen fibers; $\mathrm{G}=$ the adjacent semilunar-shaped granulation tissue. (H\&E 20×).

region. The transverse image of the inflamed sample, however, has a semilunar-shaped hypoechoic region adjacent to a hyperechoic ovoid area. In comparison with the strain field shown in Fig. 8E, the hyperechoic region corresponds to low strain values and the hypoechoic region corresponds to high strain values. The thickness of these two areas measured in the sonograms is also consistent with that estimated in strain distribution (around $2 \mathrm{~mm}$ for the hypoechoic area or the high strain region, and around $5 \mathrm{~mm}$ for the hyperechoic area or the low strain region). $\mathrm{CNR}_{\mathrm{e}}$ in this case is about $23 \mathrm{~dB}$. From the micrograph shown in Fig. 9, it is confirmed that there is semilunar-shaped granulation tissue proliferating outside of the tendon tissue. In this particular example, the sonograms agree well with the strain distribution.

Figures 10 and 11 illustrate an example (Samples $2 \mathrm{H}$ and 2I) where histology is better correlated with the estimated strain field than the sonogram. As shown in Fig. 10, the sonograms for Sample 2H (Fig. 10A and B) and 2I (Fig. 10C and D) are similar to those of healthy tendons shown previously. On the other hand, the strain field for Sample 2H has better tissue differentiation than the corresponding sonograms. Moreover, the strain variation of Sample 2I (Fig. 10F) is larger than that of Sample 2H (Fig. 10E). Defining the high strain values at $3 \mathrm{~mm}$ and $4.5 \mathrm{~mm}$ as a different tissue from that near the edges, and the high strain values at $3.5 \mathrm{~mm}$ and $5 \mathrm{~mm}$ as another different tissue from that at $3 \mathrm{~mm}$ and $4.5 \mathrm{~mm}$, the $\mathrm{CNR}_{\mathrm{e}}$ is $43 \mathrm{~dB}$ and $32 \mathrm{~dB}$, respectively. Figure 11 shows the histology of Sample 2I. The micrograph indicates that the middle area corresponds to the high strain region and is filled with granulation tissues associated with necrosis. Note that muscle tissue also exists among granulation tissues in Fig. 11. Hence, the change in tissue type may explain the high strain variation in the middle 

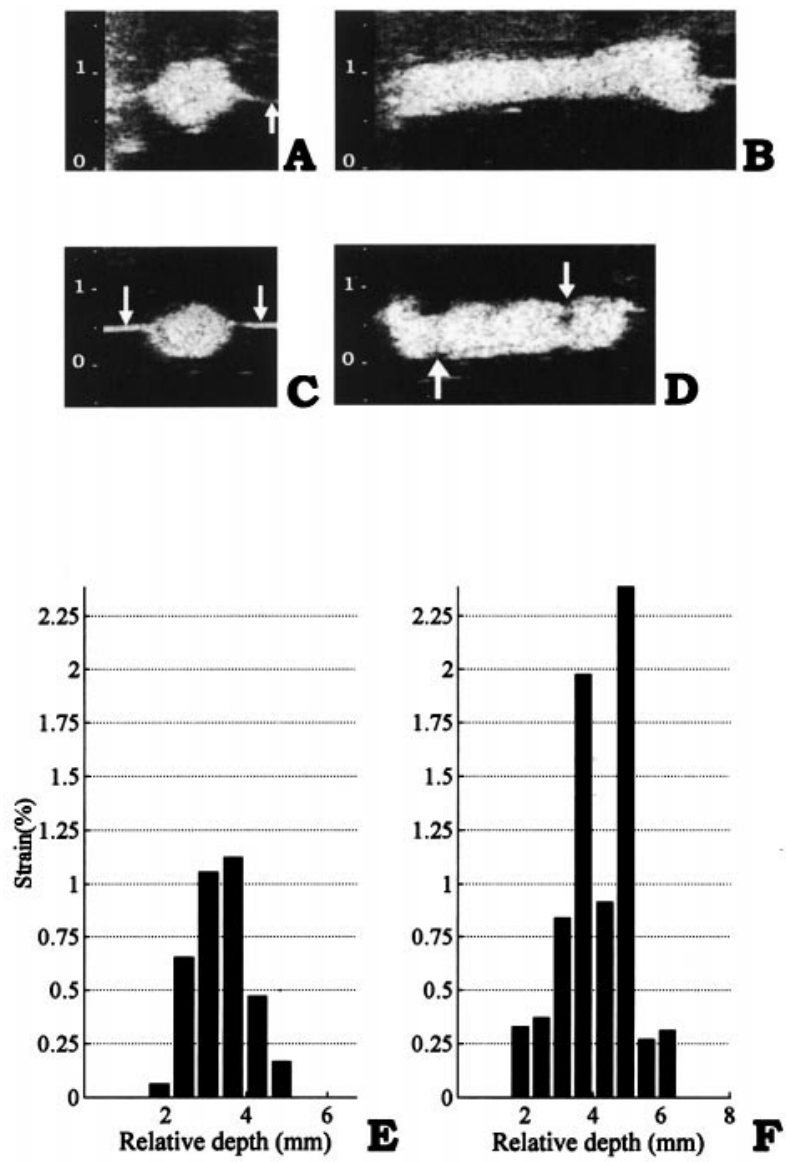

Fig. 10. B-mode sonograms and strain fields for Samples $2 \mathrm{H}$ and 2I (Pair 2, healthy and inflamed). (A) and (B) are for Sample 2H. (C) and (D) are for Sample 2I. (A) and (C) are sonograms in the transverse view; (B) and (D) are sonograms in the longitudinal view. The white arrows in (A) and (C) indicate the boundary between two layers of gelatin. The white arrows in (D) point out the suture artifacts. (E) Strain field of Sample $2 \mathrm{H}$; (F) strain field of Sample 2I.

2-mm region. The micrograph of Sample $2 \mathrm{H}$ was previously shown in Fig. 5.

\section{DISCUSSION}

In this paper, we successfully measured the axial components of transverse strain fields in rabbit Achilles tendons using the baseband speckle-tracking algorithm. Although tendons are generally harder to compress than normal soft tissues, the baseband speckle-tracking technique was able to detect displacements as small as a few microns. The strain measurements showed reasonable agreement with histology, whereas the contrast of the sonograms was sometimes insufficient for tissue differentiation. It is concluded that strain imaging has the potential to be a useful tool for evaluating tendon disorders during the rehabilitation process.

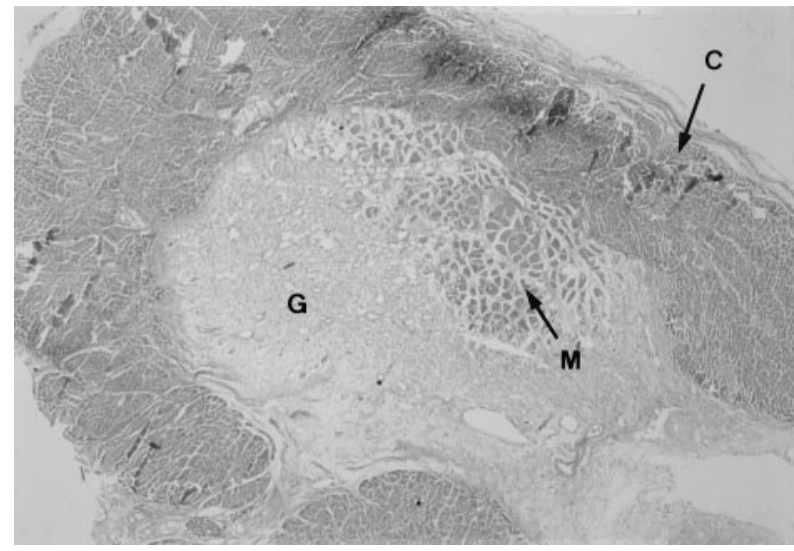

Fig. 11. Micrograph of Sample 2I (Pair 2, inflamed). G = granulation tissue proliferated within the center muscle region (M); $\mathrm{C}=$ the peripherally spreading collagen bundles. (H\&E $20 \times)$.

Although the axial components of transverse strain fields were successfully measured, reconstruction of the elastic modulus requires additional information. Ideally, the elastic modulus can be calculated numerically based on a set of linear equations made from both the static equilibrium equations and the stress-strain constitutive equations. Unfortunately, the stress component cannot be directly measured in most situations (including the experiments performed in this study). Therefore, alternative information must be provided to reconstruct the elastic modulus. Several papers in the literature have addressed these issues (Kallel and Betrand 1996; Ponnekanti et al. 1994, 1995; Skovoroda et al. 1994, 1995; Sumi et al. 1995). In general, a theoretical model for an isotropic, continuous and almost incompressible medium is formulated first. The elastic behavior of such a material can, thus, be completely characterized by the spatial distribution of either the shear modulus or the Young's modulus. Under static equilibrium conditions, the model is reduced to a closed set of coupled differential equations in terms of the displacement vector. The elastic modulus is then solved using the three-dimensional (3-D) strain fields and boundary conditions. Implementation of this approach, however, is complicated by difficulties in measuring all components of the displacement vector. Because typical ultrasonic imaging systems only acquire two-dimensional (2-D) image data, the Young's modulus must be estimated based on displacement and strain fields from a single imaging plane (i.e., axial and lateral displacements). To simplify the 3-D problem, either spatial symmetry must be assumed in the distribution of elastic modulus, or the deformation must be carefully controlled (Skovoroda et al. 1995).

Analytic solutions could be found given particular boundary conditions and distribution of the elastic mod- 
ulus. For example, Goodier (1933) derived an analytic solution for a compressible, uniformly deformed infinite medium with a spherical or cylindrical elastic inclusion embedded in the medium. Assuming plane strain state conditions, the 3-D problem can be reduced to a 2-D problem and analytic solutions for a circularly or linearly bonded inclusion can be found (Honein and Herrmann 1990; Kallel et al. 1996). In the case of plane strain state, the out-of-plane displacement is either zero or relatively small and the in-plane displacements do not vary significantly as a function of the out-of-plane coordinate. This model is usually used to investigate large cylindrical bodies with a uniform surface displacement along the long axis of the cylinder (Kallel et al. 1996; Skovoroda et al. 1995). By using the aforementioned approaches, distribution of relative Young's modulus can be reconstructed. Furthermore, the absolute Young's modulus in the region of interest can be obtained as long as the Young's modulus of the referred medium is known.

Although such alternative techniques have been successfully verified, they are only applicable to isotropic media. Due to the anisotropic nature of tendons, new reconstruction algorithms must be developed. For example, Levison (1987) has verified that a transversely isotropic model provided a better fit of elastic constants for muscle than an isotropic model. In addition, future work is focused on modifying the measurement setup to facilitate the elastic modulus reconstruction. The new system is capable of directly estimating the elastic modulus of small samples (i.e., without putting the sample in a gelatin-based phantom). The irregular tendon geometry is also being considered and finite element analysis will be used to investigate the performance of the new system.

Tendon inflammation can be classified into four categories: paratenonitis (also known as tenosynovitis), paratenonitis with tendinosis, tendinosis and tendinitis (Leadbetter 1994). As the tendon recovers, tendinitis can be further divided into four stages: necrosis stage, proliferate stage, remodeling stage, and maturation stage. During recovery, the damaged collagen fibers are repaired to regain strength and stiffness while the granulation tissue gradually condenses to scar tissue and decreases its cellularity. Although the experiments in this study were only designed to simulate tendon inflammation at an early stage, it is expected that elasticity imaging may be a useful tool to monitor tendon recovery. This will be verified by future experiments with improved experiment protocols.

In this study, tendons were suspended in a gelatinbased phantom for in vitro strain measurements. For in vivo measurements, however, the tendon is constrained by connections to bone and muscle. The complex geometry of neighboring organs (i.e., adjacent tendons, ves- sels, bone, ... etc.) may complicate the mechanical boundary conditions. Because tendons are anisotropic, 3-D image data may be required to determine the 3-D displacement field. On the other hand, because most tendons are close and parallel to the skin surface, the applied surface deformation may be easier to be perpendicular to the long axis of the tendon and the required deformation may be smaller than that for deep-lying organs such as liver and kidney. With small surface displacements, the linear elasticity model becomes more applicable. In addition, most of the tendons in the limbs exhibit a long cylindrical shape, which may make it easier to create plane strain state.

In conclusion, we have verified that the axial components of the tendon's transverse strain fields can be measured by US using the baseband speckle-tracking algorithm. We have also correlated the histology of the tendon with its strain distribution. Compared to conventional B-mode imaging, strain distribution may be a better tool for clinical evaluation of tendon disorders. Future works will focus on reconstruction of elastic modulus of inflamed tendons throughout the complete recovery process.

Acknowledgements-This work was supported in part by NSC Grant NSC-87-2218-E-002-052. The authors also thank Drs. C-L. Wang, J-Y. Shieh and T-G. Wang of National Taiwan University Hospital for help in sonogram evaluation, and Dr. T-T. Wu of the Institute of Applied Mechanics for supporting materials for experiments. Finally, the authors greatly appreciate insightful comments made by the reviewers.

\section{REFERENCES}

Bilgen M, Insana MF. Predicting target detectability in acoustic elastography. IEEE Ultrason Sympos 1997;2:1427-1430.

Cespedes I, Ophir J. Reduction of image noise in elastography. Ultrason Imag 1993;15:89-102.

Cespedes I, Ophir J. Ponnekanti H, Maklad N. Elastography: elasticity imaging using ultrasound with application to muscle and breast in vivo. Ultrason Imag 1993;15:73-88.

Fornage BD. Musculoskeletal ultrasound. New York: Churchill Livingstone, 1995

Fung YC. Biomechanics: mechanical properties of living tissues. New York: Springer-Verlag, 1993:242-310.

Garra BS, Cespedes I, Ophir J, et al. Elastography of breast lesion: initial clinical results. Radiology 1997;202:79-86.

Goodier JN. Concentration of stress around spherical and cylindrical inclusions and flaws. Trans ASME 1933;55:39-44.

Holsbeeck MV, Introcaso JH. Musculoskeletal ultrasound. St. Louis: Mosby-Year Book, 1991.

Honein T, Herrmann G. On bonded inclusions with circular or straight boundaries in plane elastostatics. Trans ASME J Appl Mech 1990; 57:850-856.

Insana MF, Bilgen M, Chaturvedi P, Hall TJ, Bertrand M. Signal processing strategies in acoustic elastography. IEEE Ultrason Sympos 1996;2:1139-1142.

Kaisar S, Ophir J. Reduction of signal decorrelation from mechanical compression of tissue by temporal stretching: applications to elastography. Ultrasound Med Biol 1997;23(1):95-105.

Kallel F, Bertrand M. Tissue elasticity reconstruction using linear perturbation method. IEEE Trans Med Imag 1996;15(3):209-213.

Kallel F, Bertrand M, Ophir J. Fundamental limitations on the contrasttransfer efficiency in elastography: an analytic study. Ultrasound Med Biol 1996;22(4):463-470. 
Kallel F, Ophir J, Magee K, Krouskop T. Elastographic imaging of low-contrast modulus distributions in tissue. Ultrasound Med Biol 1998;24(3):409-425.

Kallel F, Varghese T, Ophir J, Bilgen M. The nonstationary strain filter in elastography: part II. lateral and elevational decorrelation. U1trasound Med Biol 1997;23(9):1357-1369.

Krouskop TA, Levison SF. A pulsed Doppler ultrasonics system for making noninvasive measurements of the mechanical properties of soft tissue. J Rehabil Res Dev 1987;24:1-8.

Leadbetter WB. Soft tissue athletic injury. In: Fu FH, Stone DA, eds. Sports injuries: mechanisms, prevention, and treatment. Baltimore: Williams \& Wilkins, 1994:733-780.

Lerner RM, Huang SR, Parker KJ. "Sonoelasticity" images derived from ultrasound signals in mechanically vibrated tissues. Ultrasound Med Biol 1990;16(3):231-239.

Levison SF. Ultrasound propagation in anisotropic soft tissue: the application of linear elasticity theory. J Biomech 1987;20(3):251-260.

Levison SF, Shinagawa M, Sato T. Sonoelastic determination of human skeletal muscle elasticity. J Biomech 1995;28(10):1145-1154.

Marcelis S. Peripheral musculoskeletal ultrasound atlas. New York: Theme Medical Publishers, 1996.

Mason ML, Allen HS. The rate of healing of tendons: a experimental study of tensile strength. Ann Surg 1941;113:424-459.

O’Donnell M, Skovoroda AR, Shapo BM, Emelianov ST. Internal displacement and strain imaging using ultrasonic speckle tracking. IEEE Trans Ultrason Ferroelec Freq Control 1994;41(3):314-325.

Ophir J, Cespedes I, Ponnekanti H. Elastography: a quantitative method for imaging the elasticity of biological tissues. Ultrason Imag 1991;13:111-134.

Ophir J, Kallel F, Varghese T, et al. Elastography: a systems approach. Int J Imaging Syst Technol 1997;8:89-103.

Ponnekanti H, Ophir J, Cespedes I. Ultrasonic imaging of the stress distribution in elastic media due to an external compressor. Ultrasound Med Biol 1994;20(1):27-33.

Ponnekanti H, Ophir J, Huang Y, Cespedes I. Fundamental mechanical limitations on the visualization of elasticity contrast in elastography. Ultrasound Med Biol 1995;21(4):533--543.
Sarvazyan AP. Method and device for shear wave elasticity imaging. US Patent Number 5,606,971, 1997.

Skovoroda AR, Emelianov ST, Lubinski MA, et al. Theoretical analysis and verification of ultrasound displacement and strain imaging. IEEE Trans Ultrason Ferroelec Freq Control 1994; 41(3):302-313.

Skovoroda AR, Emelianov ST, O’Donnell M. Tissue elasticity reconstruction based on ultrasonic displacement and strain images. IEEE Trans Ultrason Ferroelec Freq Control 1995;42(4):747-765.

Sumi C, Nakayama K, Suzuki A. Estimation of shear modulus distribution in soft tissue from strain distribution. IEEE Trans Biomed Eng 1995;42:193-202.

Varghese T, Ophir J. Estimation tissue strain from signal decorrelatin using correlation coefficient. Ultrasound Med Biol 1996;22(9): 1249-1254.

Varghese T, Ophir J. A theoretical framework for performance characterization of elastography: the strain filter. IEEE Trans Ultrason Ferroelec Freq Control 1997a;44(1):164-172.

Varghese T, Ophir J. Enhancement of echo-signal correlation in elastography using temporal stretching. IEEE Trans Ultrason Ferroelec Freq Control 1997b;44(1):173-180.

Varghese T, Ophir J. The nonstationary strain filter in elastography: part I. frequency dependent attenuation. Ultrasound Med Biol 1997c;23(9):1343-1356.

Varghese T, Ophir J. An analysis of elastographic contrast-to-noise ratio. Ultrasound Med Biol 1998;24(6):915-924.

Varghese T, Ophir J, Cespedes I. Noise reduction in elastograms using temporal stretching with multicompression averaging. Ultrasound Med Biol 1996;22(8):1043-1052.

Yamakoshi Y. Ultrasonic imaging of internal vibration of soft tissue under forced vibration. IEEE Trans Ultrason Ferroelec Freq Control 1990; 17:45-53.

Yeung F, Levison SF, Parker KJ. Multilevel and motion model-based ultrasonic speckle tracking algorithms. Ultrasound Med Biol 1998; 24(3):427-441. 